\title{
White matter integrity and processing speed in sickle cell anemia
}

Hanne Stotesbury, MSc, Fenella J. Kirkham, MD Res, Melanie Kölbel, MSc, Philippa Balfour, MSc, Jonathan D. Clayden, PhD, Sati Sahota, BSc, Simrat Sakaria, MSc, Dawn E. Saunders, MD Res, Jo Howard, MD Res, Rachel Kesse-Adu, FRCPath, Baba Inusa, FRCPCH, Maria Pelidis, MD, Subarna Chakravorty, FRCPCH, David C. Rees, MD Res, Moji Awogbade, FRCPath, Olu Wilkey, FRCPCH, Mark Layton, FRCP, Christopher A. Clark, PhD, and Jamie M. Kawadler, PhD

Neurology ${ }^{\circledR}$ 2018;90:e2042-e2050. doi:10.1212/WNL.0000000000005644

\section{Abstract}

\section{Objective}

The purpose of this retrospective cross-sectional study was to investigate whether changes in white matter integrity are related to slower processing speed in sickle cell anemia.

\section{Methods}

Thirty-seven patients with silent cerebral infarction, 46 patients with normal MRI, and 32 sibling controls (age range 8-37 years) underwent cognitive assessment using the Wechsler scales and 3-tesla MRI. Tract-based spatial statistics analyses of diffusion tensor imaging (DTI) and neurite orientation dispersion and density imaging (NODDI) parameters were performed.

\section{Results}

Processing speed index (PSI) was lower in patients than controls by 9.34 points (95\% confidence interval: 4.635-14.855, $p=0.0003$ ). Full Scale IQwas lower by 4.14 scaled points (95\% confidence interval: -1.066 to $9.551, p=0.1$ ), but this difference was abolished when PSI was included as a covariate $(p=0.18)$. There were no differences in cognition between patients with and without silent cerebral infarction, and both groups had lower PSI than controls (both $p<0.001)$. In patients, arterial oxygen content, socioeconomic status, age, and male sex were identified as predictors of PSI, and correlations were found between PSI and DTI scalars (fractional anisotropy $r=0.614, p<0.00001 ; r=-0.457, p<0.00001$; mean diffusivity $r=-0.341, p=0.0016$; radial diffusivity $r=-0.457, p<0.00001$ ) and NODDI parameters (intracellular volume fraction $r=0.364, p=0.0007$ ) in widespread regions.

\section{Conclusion}

Our results extend previous reports of impairment that is independent of presence of infarction and may worsen with age. We identify processing speed as a vulnerable domain, with deficits potentially mediating difficulties across other domains, and provide evidence that reduced processing speed is related to the integrity of normal-appearing white matter using microstructure parameters from DTI and NODDI.

\author{
Correspondence \\ Dr. Kirkham \\ Fenella.Kirkham@ucl.ac.uk
}




\section{Glossary}

$\mathbf{C a O}_{2}=$ arterial oxygen content $;$ DTI = diffusion tensor imaging; FA = fractional anisotropy; FLAIR = fluid-attenuated inversion recovery; FSIQ = Full Scale IQ; FSL = FMRIB's Software Library; ICVF = intracellular volume fraction; MD = mean diffusivity; NODDI = neurite orientation dispersion and density imaging; ODI = orientation dispersion index; POMS = Prevention of Morbidity in Sickle Cell Disease; PSI = processing speed index; RD = radial diffusivity; SAC = Sleep and Asthma Cohort followup study; SCA = sickle cell anemia; $\mathbf{S C I}=$ silent cerebral infarction; $\mathbf{S E S}=$ socioeconomic status; $\mathbf{S p o}_{2}=$ daytime oxygen saturation; TE = echo time; TR = repetition time; WAIS = Wechsler Adult Intelligence Scale; WASI = Wechsler Abbreviated Scale of Intelligence; WISC $=$ Wechsler Intelligence Scale for Children

Even in the absence of silent cerebral infarction (SCI), patients with sickle cell anemia (SCA) are at risk of cognitive impairment that may worsen with age ${ }^{1,2}$ and affect quality of life. ${ }^{3}$ Reduced processing speed is the most prominent impairment $^{4}$ and may mediate difficulties across other domains, ${ }^{5}$ but the etiology is not well understood, and there are no models of risk factors.

MRI studies have revealed hemodynamic ${ }^{6-11}$ and structural abnormalities $^{12-14}$ that may underlie cognitive impairment. Diffusion tensor imaging (DTI) studies have reported widespread reductions in fractional anisotropy (FA) and increases in radial diffusivity $(\mathrm{RD}){ }^{15-17}$ Diffusion changes have been associated with oxygen desaturation and anemia ${ }^{15}$ and may relate to reduced processing speed, but functional consequences have yet to be investigated.

A limitation of DTI is that the parameters are not specific to particular microstructural elements of white matter. Neurite orientation dispersion and density imaging (NODDI) ${ }^{18}$ may offer more sensitivity and specificity as it models changes in fiber dispersion (orientation dispersion index [ODI]) as well as density of the tissue microstructure (intracellular volume fraction [ICVF]). NODDI has been successfully applied in studies of typical development ${ }^{19,20}$ and clinical populations ${ }^{21}$ but not yet in SCA.

In the present study, we aimed to (1) investigate differences in processing speed between patients with SCA, grouped by presence of SCI, and controls; (2) explore the effect of processing speed on general intelligence and potential risk factors for deficits; and (3) examine the relationship between DTI and NODDI-derived indices of white matter microstructure and processing speed.

\section{Methods}

\section{Patients}

Patients were aged 8 to 38 years and enrolled in 2 studies at University College London: the Sleep and Asthma Cohort follow-up study (SAC-III) ${ }^{22}$ and Prevention of Morbidity in SCA 2b (POMS) ${ }^{23}$ baseline investigation. Controls were healthy siblings of patients recruited to either study with no history of neurologic or psychiatric conditions. Participants were recruited and assessed between 2015 and 2016. Patients were ineligible for SAC and POMS study participation if they were receiving nocturnal respiratory support at the time of enrollment, participating in a clinical trial evaluating blood transfusion or oxygen therapy, or had chronic lung disease (other than asthma) or existing respiratory failure. Additional exclusion criteria for the POMS study were hospital admissions for acute sickle complications within 1 month of enrollment, more than 6 hospital admissions for acute sickle complications within 12 months of enrollment, overnight oximetry showing mean overnight saturation of less than $90 \%$ for more than $30 \%$ of total sleep time, severe sleep apnea defined by $4 \%$ oxygen desaturation index $>15 / \mathrm{h}$, and chronic blood transfusion or transfusion within 3 months of enrollment. For the SAC study, patients were enrolled without regard to past sickle- or sleep-related morbidity or transfusion status.

\section{Standard protocol approvals, registrations, and patient consents}

Ethical approval was granted by West London and South Yorkshire research ethics committees, respectively. Full informed consent and assent according to the Declaration of Helsinki were obtained from participants and for children from their parent/guardian.

\section{Cognitive variables}

Full Scale IQ (FSIQ) was measured using the Wechsler Abbreviated Scale of Intelligence (WASI-II subscale IQ; POMS patients), Wechsler Intelligence Scale for Children (WISCIV; SAC patients and controls younger than 16 years), or the Wechsler Adult Intelligence Scale (WAIS-IV; SAC patients and controls 16 years or older). Processing speed index (PSI) was derived from the WISC-IV or the WAIS-IV using the coding and symbol search subtests. Strong correlations have been demonstrated between editions (WASI/WAIS/WISC) and between the child and adult versions (WISC/WAIS), justifying their inclusion in the same analyses. ${ }^{24,25}$ Assessments were double-scored by trained assessors (J.M.K., M.K., H.S., P.B.) that were blinded to disease status. In the event of disagreement or ambiguity, the opinion of a third assessor was sought.

\section{Socioeconomic variables}

Education decile was obtained from UK postcode to provide an index of socioeconomic status (SES). ${ }^{26}$ This scale captures attainment and skills in local areas based on several indicators: 
average scores for pupils in state-funded schools at ages 7-11 and 14-16 years, absence from state-funded secondary schools, proportion of people staying on in education/ training post 16 years, entry to higher education, proportion of working adults with no/low qualifications and language proficiency. Total scores are ranked from 1 to 10 , with 1 representing the most deprived.

\section{Hematologic variables}

Steady-state hemoglobin was recorded from patient medical records using the closest available full blood within 6 months of the day of cognitive testing. Arterial oxygen content $\left(\mathrm{CaO}_{2}\right)$ was calculated using:

$$
\mathrm{CaO}_{2}=1.34 \times \text { Hemoglobin } \times \text { Oxygen saturation }+.003 \times \mathrm{pO}_{2}
$$

where oxygen saturation $\left(\mathrm{SpO}_{2}\right)$ was estimated by pulse oximetry on the day of cognitive testing (SAC) or the baseline clinic visit (POMS), and $\mathrm{pO}_{2}$, the partial pressure of oxygen, was assumed to be 100 torr in room air.

\section{MRI acquisition}

Imaging was conducted within 2 weeks of cognitive assessment on a $3 \mathrm{~T}$ Siemens Prisma (Erlangen, Germany) with $80 \mathrm{mT} / \mathrm{m}$ gradients and a $64-$ channel receive head coil. The MRI protocol included axial T2-weighted (repetition time $[\mathrm{TR}]=8,420$ milliseconds $[\mathrm{ms}]$, echo time $[\mathrm{TE}]=68 \mathrm{~ms}$, voxel size $=0.51 \times 0.51 \times 5.6 \mathrm{~mm})$, fluid-attenuated inversion recovery (FLAIR) $(\mathrm{TR}=5,000 \mathrm{~ms}, \mathrm{TE}=395 \mathrm{~ms}$, voxel size $=0.65 \times 1 \times 0.65 \mathrm{~mm})$, and diffusion-weighted $\left(\mathrm{TR}=3,050 \mathrm{~ms}, \mathrm{TE}=60 \mathrm{~ms}, 2\right.$ shells at $\mathrm{b}=1,000 \mathrm{~s} / \mathrm{mm}^{2}$ and $\mathrm{b}=2,200 \mathrm{~s} / \mathrm{mm}^{2}$ with 13 interleaved $\mathrm{b}=0$ images, voxel size $=$ $2 \times 2 \times 2 \mathrm{~mm}$ ) sequences. A neuroradiologist (D.S.), blinded to disease status, read each participant's MRI and classified SCI according to the criteria of a hyperintensity on FLAIR of more than $3 \mathrm{~mm}$ in diameter and present on 2 planes, as for the Silent Infarction Transfusion trial. ${ }^{27}$

\section{MRI processing}

The diffusion images were preprocessed using TractoR 3.0.7 ${ }^{28}$ and FSL 5.0.1. ${ }^{29}$ Images were visually screened for motion and corrected for susceptibility-induced distortions and eddy current artifact using FSL. Maps for each of the DTI parameters were generated in FSL by fitting a diffusion tensor model to each voxel using a weighted least-squares method. ODI and ICVF maps were generated using the NODDI MATLAB Toolbox. ${ }^{18}$ DTI and NODDI parameters were analyzed using whole-brain, voxel-wise tract-base spatial statistics. The specifics of this approach have been described elsewhere. $^{30}$ Briefly, each participant's FA map was aligned with every other FA map, and the most representative map was used as the target. The target was affine-aligned to Montreal Neurological Institute standard space. All FA maps underwent nonlinear transformation to the target and affine transformation to standard space. FA maps were merged, and voxels with the highest FA at the core of main white matter tracts (threshold: $\mathrm{FA}=0.2$ ) were used to create a mean FA skeleton. Each participant's FA map was projected onto the mean FA skeleton, enabling voxel-wise statistical analyses. The maps for the remaining parameters were similarly projected onto the skeleton for analyses. Reference was made to the JHU (Johns Hopkins University) DTI white matter atlas to describe the locations of significant voxels. ${ }^{31}$

\section{Statistical analysis}

Analyses were performed in RStudio Desktop 1.0.153 using the companion to applied regression ${ }^{32}$ and global validation of linear models ${ }^{33}$ packages. Prior to statistical analysis, neurocognitive variables were assessed for normality and equality of variance using the Shapiro-Wilk and Levene tests, respectively. For all analyses, results were considered significant at $p<0.05$. FSIQ and PSI were compared between patient and sibling control groups using type II analyses of covariance including education deciles as covariates. The effect of PSI on other domains of cognition was explored by including PSI as a covariate in comparisons between patients and controls in FSIQ. An exploratory multiple linear regression analysis was performed to predict PSI from previously implicated and potentially confounding variables: presence of $\mathrm{SCI}(\mathrm{SCI}+/-), \mathrm{CaO}_{2}$, education decile, age, sex, hydroxyurea use, and transfusion status. Influential measures were assessed by calculating the standardized difference of the $\beta$ for each model variable, difference in fits, covariance ratios, Cook distances, and the diagonal elements of the hat matrix. For all patients, intersubject voxel-wise correlation was performed between DTI and NODDI parameters and PSI, while treating age, sex, and postcode-based education deciles as covariates. Threshold-free cluster enhancement was used to correct for multiple comparisons.

\section{Data availability}

Full anonymized data will be shared at the request from any qualified investigator. Interactive maps from imaging analyses will be uploaded on neurovault where results can be explored and downloaded (neurovault.org/collections/3510/).

\section{Results}

\section{Patient characteristics}

There were no differences between groups in age or sex (table 1). Of 83 patients ( 82 sickle cell hemoglobin, 1 hemoglobin $S / \beta^{0}$-thalassemia), 37 (45\%) without neurologic signs were identified with SCI (SCI+). Lesions were rightsided in 8 patients, left-sided in 6 , and bilateral in 23. Thirteen patients had lesions in more than one region; 35 had frontal lesions, 12 had parietal lesions, 2 had temporal lesions, and 3 had occipital lesions. Lesions were most frequently located in the border zones between arterial distributions in the deep frontal white matter. Twenty-nine patients were on hydroxyurea (16 SCI-), 5 were on chronic transfusions (3 SCI-), and 9 had undergone a transfusion within 6 months of assessment (6 SCI-). Mean hemoglobin, $\mathrm{SpO}_{2}$, and $\mathrm{CaO}_{2}$ were lower than reference norms (table 1). Eighteen patients (22\%, 8 SCI-) were desaturated with $\mathrm{SpO}_{2} \leq 96 \%$. Mean hemoglobin and $\mathrm{CaO}_{2}$ were lower in patients with SCI, but there were no 
Table 1 Sample demographics and cognitive performance

\begin{tabular}{|c|c|c|c|c|c|}
\hline & Controls $(n=32)$ & $S C I-(n=46)$ & $\mathrm{SCl}+(\mathrm{n}=37)$ & Inferential statistics & Post hoc \\
\hline Median age, y & $15.26(8-30)$ & $14.62(8-37)$ & $16.34(8-36)$ & - & - \\
\hline Sex, males, n (\%) & $14(43)$ & $23(50)$ & $20(54)$ & - & - \\
\hline Socioeconomic status & $4.94(2.26)$ & $5.370(2.26)$ & $5.11(1.97)$ & $F_{2,112}=0.398, p=0.6723$ & NS \\
\hline Full Scale IQ & $96.50(11.62)$ & $93.72(12.99)$ & $90.68(13.52)$ & $F_{2,111}=1.799, p=0.1702$ & NS \\
\hline Processing speed index & $97.81(11.32)$ & 90.07 (14.28) & $86.49(11.26)$ & $F_{2,111}=7.876, p=0.0006^{c}$ & $--^{a, b}$ \\
\hline Hemoglobin, g/L & - & $90.96(13.66)$ & $84.54(12.92)$ & $t_{78.839}=2.192, p=0.0313^{c}$ & - \\
\hline Oxygen saturation & - & $97.37(1.95)$ & $96.46(3.08)$ & $t_{58.096}=1.564, p=0.1233$ & - \\
\hline Oxygen content, $\mathrm{mL} / \mathrm{dL}$ & - & $12.17(1.79)$ & $11.25(1.87)$ & $t_{78.839}=2.192, p=0.03134^{c}$ & - \\
\hline
\end{tabular}

Abbreviations: NS = not significant; $\mathrm{SCl}-=$ patients without silent cerebral infarction; $\mathrm{SCl}+=$ patients with cerebral infarction.

Values are summary and test statistics.

${ }^{a}$ Controls $>\mathrm{SCl}+$.

${ }^{b}$ Controls $>\mathrm{SCl}-$.

'Statistically significant.

differences in saturation (figure 1). This pattern remained when transfused patients were excluded.

\section{Neurocognitive variables}

The data met the analyses of covariance and multiple linear regression assumptions. After controlling for the effect of education deciles, mean PSI was lower in patients than controls by 9.34 scaled points $(95 \%$ confidence interval: 4.635-14.855, $p=0.0003)$. FSIQ was lower by 4.14 scaled points ( $95 \%$ confidence interval: -1.066 to $9.551, p=0.1$ ), but this difference was abolished when PSI was included as a covariate $(p=0.18)$. There were no differences in cognition between patients with and without SCI, and both groups had lower PSI (table 1; $p<0.001$ ) but not FSIQ, compared to controls (figure 1). This pattern remained when analyses were repeated in children and adults separately $(p<0.05)$.

Multiple linear regression was conducted to predict PSI from previously implicated variables. Of all the predictors, only male sex, $\mathrm{CaO}_{2}$, and education decile (SES) had zero-order correlations with PSI (figure 2). However, in the full model, all predictors apart from SCI and chronic transfusion had partial effects (table 2). The 7-predictor model was able to account for $25 \%$ of the variance in PSI $\left(F_{8,74}=3.042, p=\right.$ 0.005 , multiple $\left.R^{2}=0.25\right)$. All predictors remained when 11 influential cases, including all 5 patients on chronic transfusion, were removed from the analysis $\left(F_{7,64}=3.404, p=\right.$ 0.004 , multiple $R^{2}=0.27$ ).

\section{Neuroimaging metrics}

In patients, PSI was correlated with FA, MD, RD, and ICVF (figure 3). Specifically, decreases in PSI were associated with decreases in FA across the internal capsule and corpus callosum $\left(r_{81}=0.614, p<0.00001,30,492\right.$ voxels $)$, and with decreases in ICVF in more widespread regions covering much of the white matter skeleton, with clusters extending throughout the corpus callosum, corona radiata, and superior and inferior longitudinal fasciculi $\left(r_{81}=0.364, p=0.0007\right.$, 70,659 voxels). In addition, decreases in PSI were associated with increases in $\mathrm{MD}\left(r_{81}=-0.341, p=0.0016,82,663\right.$ voxels) and $\mathrm{RD}$, also in widespread regions, with many clusters located posteriorly, including the posterior corona radiata and splenium of the corpus callosum, respectively $\left(r_{81}=\right.$ $-0.457, p<0.00001,67,296$ voxels). Statistical maps can be viewed and explored interactively at neurovault.org/collections/3510/. These correlations remained when examined in $\mathrm{SCI}+$ and SCI- groups separately. There were no relationships between PSI and axial diffusivity or ODI.

\section{Discussion}

This study provides evidence for a relationship between reductions in processing speed and changes in DTI and NODDI parameters in SCA and models risk factors. Patients with SCA showed processing speed deficits, irrespective of presence of SCI. The results suggest that the degree of slower processing speed is related to loss of white matter integrity, and that lower $\mathrm{CaO}_{2}$ and SES may be independent risk factors for deficit.

PSI was lower in patients than controls by 9 scaled points. FSIQ was numerically lower by 4 scaled points, but this difference did not reach significance. Differences in PSI were greater than the often-cited 7-point (one-half of an SD) threshold for clinically meaningful differences. ${ }^{34}$ Moreover, although mean PSI in the patient group fell in the low-average range, $28 \%$ of patients had PSI scores that fell in the borderline to extremely low ranges (i.e., scores of $<80$ ) compared to $6 \%$ of controls. Taken together, these results suggest that although there is variability within the SCA population, patients with SCA are at risk of clinically significant cognitive difficulties. Controlling for PSI abolished a numerical 
A

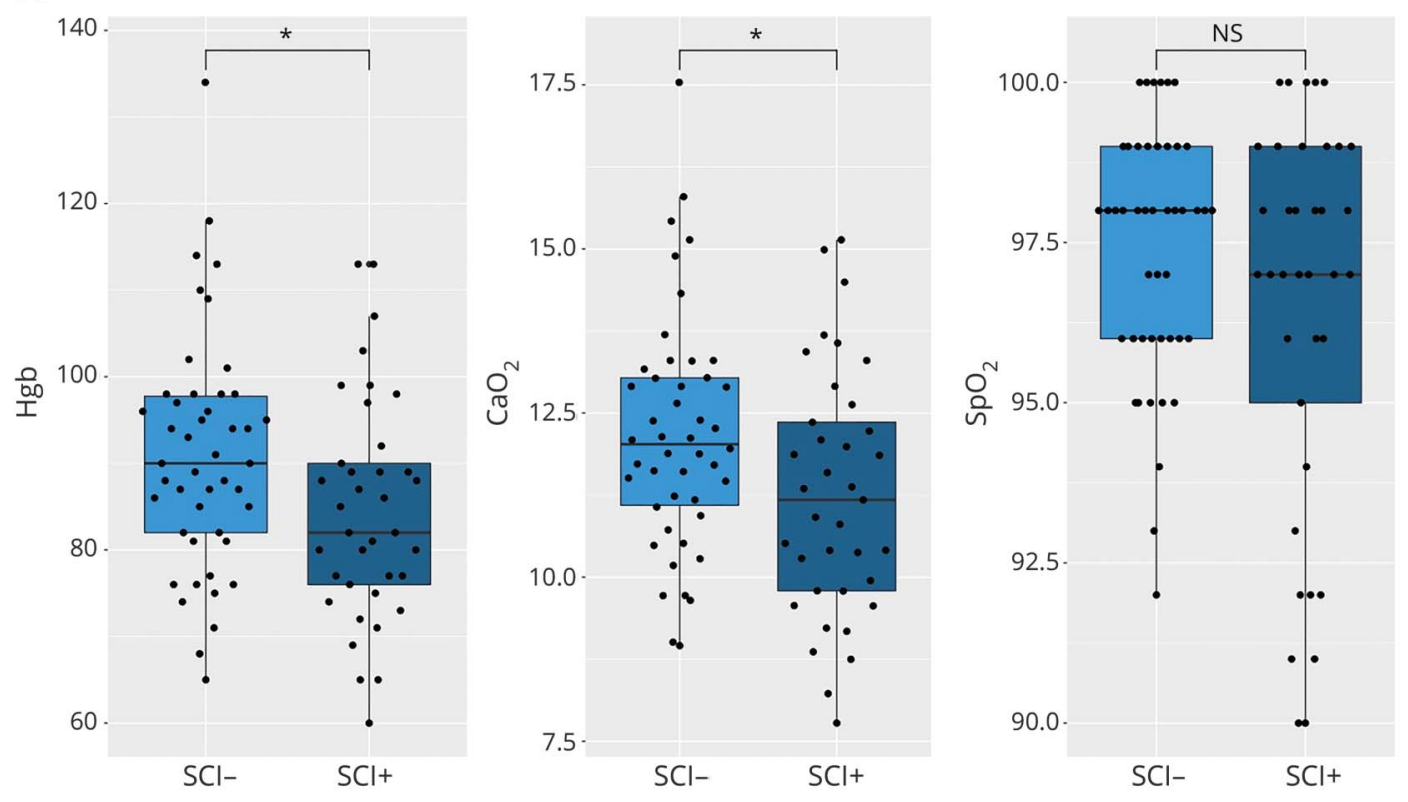

B

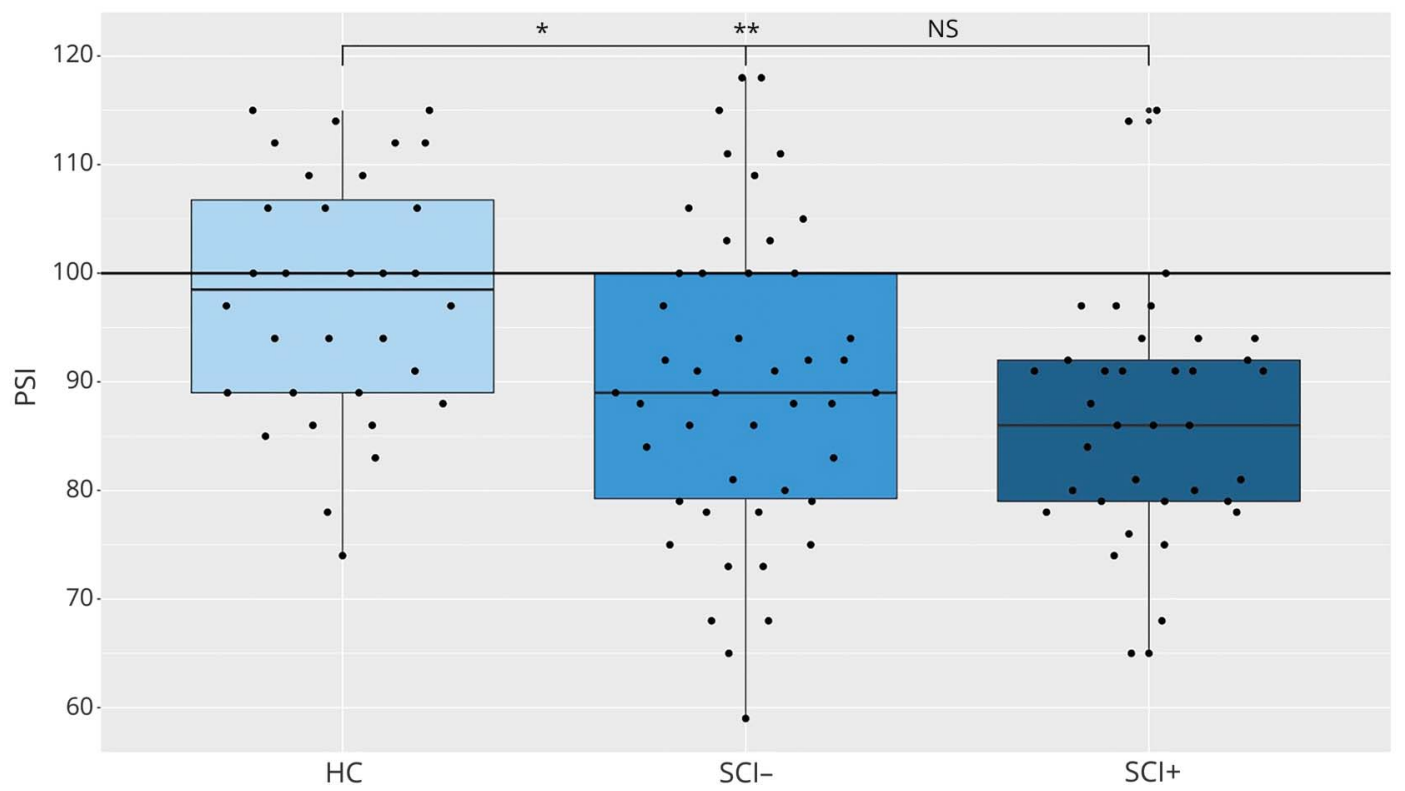

(A) Differences in hemoglobin ( $\mathrm{Hgb}$; left), arterial oxygen content ( $\mathrm{CaO}_{2}$; middle), and oxygen saturation ( $\left.\mathrm{SpO}_{2}\right)$, between patients with ( $\mathrm{SCl}+$ ) and without ( $\mathrm{SCl}-$ ) silent cerebral infarction. (B) Differences in processing speed index (PSI) between healthy controls (HCS) and patients (SCI-, $\mathrm{SCl}+$ ). * $p<0.05$; $* * p<0.01$ (after Bonferroni correction for multiple comparisons). Horizontal line represents mean PSI in the normative population. NS = not significant.

difference between patients and controls in FSIQ. These findings extend those of studies with adults ${ }^{5}$ to children with SCA and are consistent with the notion that slower processing may contribute to other cognitive difficulties. Research on aging in the general population has similarly highlighted that fast and efficient information processing may be a prerequisite for higher-order cognitive abilities. ${ }^{35}$

Although cognitive performance was scaled for age, age was a negative predictor of PSI in our regression model. PSI has yet to be examined longitudinally in SCA, but this finding is in agreement with previous reports and may suggest worsening cognitive function with age in SCA. ${ }^{1,4,36}$ While processing speed has been shown to predict academic attainment in typical development, $^{37}$ further work is required to examine the developmental trajectory of PSI in SCA and to investigate the effect of deficits on abilities potentially important for life outcomes.

In patients, after correcting for the effects of age and sex, strong correlations were found between PSI and multiple diffusion-derived indices of white matter microstructure. Correlated regions were widespread, and only partially 
Figure 2 Correlations between predictors of processing speed

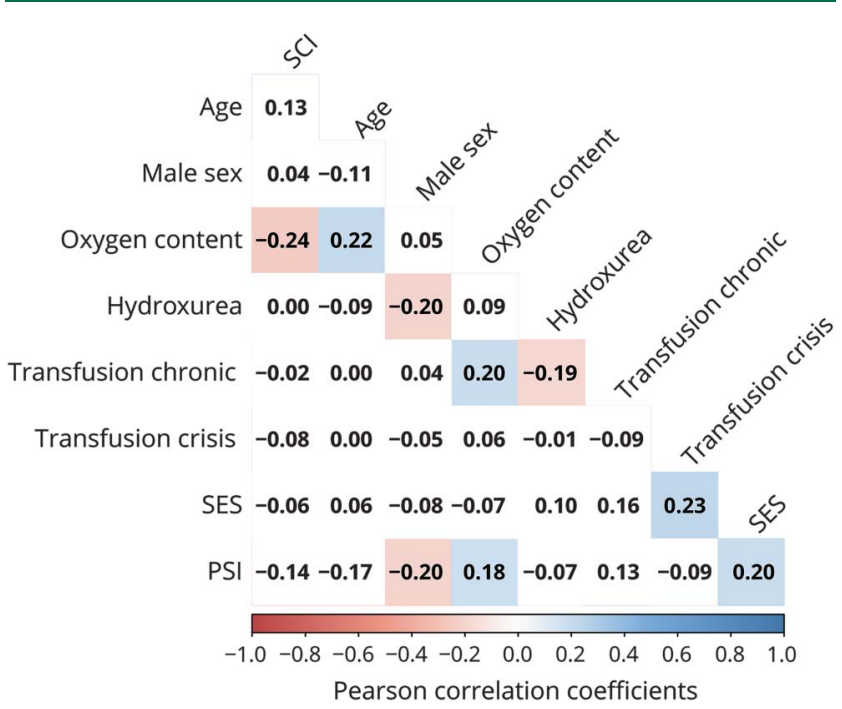

Correlogram visualizing relationships between variables included in the exploratory regression analysis. Values are zero-order Pearson correlation coefficients. Shaded areas represent significant relationships. Blue colors represent positive relationships, whereas red colors represent negative relationships. Intensity signifies the strength of relationships. PSI = processing speed index; $\mathrm{SCl}=$ silent cerebral infarction; $\mathrm{SES}$ = socioeconomic status.

overlapped with lesions, suggesting that slower processing speed is related to the integrity of normal-appearing white matter in SCA. These results provide evidence that cognitive impairment in SCA is related to white matter integrity using quantitative microstructure parameters from multishell diffusion MRI, and highlight the utility of novel diffusion

Table 2 Regression coefficients for variables predicting processing speed index

\begin{tabular}{llc}
\hline Variable & $\boldsymbol{\beta}$ & $\mathbf{b}$ \\
\hline $\mathbf{S C l}$ & -0.009 & -0.229 \\
\hline Age & $-0.301^{\mathrm{a}, \mathrm{d}}$ & -0.649 \\
\hline Male sex & $-0.278^{\mathrm{a}, \mathrm{d}}$ & -7.228 \\
\hline $\mathrm{CaO}_{2}$ & $0.311^{\mathrm{a}, \mathrm{d}}$ & 2.172 \\
\hline SES & $0.283^{\mathrm{b}, \mathrm{d}}$ & 1.734 \\
\hline Hydroxyurea & $-0.211^{\mathrm{c}}$ & -5.760 \\
\hline Tx crisis & $-0.190^{\mathrm{c}}$ & -7.955 \\
\hline Tx chronic & -0.020 & -1.092 \\
\hline
\end{tabular}

Abbreviations: $\mathrm{CaO}_{2}=$ arterial oxygen content; $\mathrm{SCl}=$ silent cerebral infarction; SES = socioeconomic status; $T x=$ transfusion.

Values are standardized regression coefficients $(\beta)$ and unstandardized regression coefficients (b) from the exploratory multiple linear regression analysis.

a $p<0.01$.

${ }^{\mathrm{b}} p<0.5$.

c $p \leq 0.1$.

d Stastically significant at $p<0.05$. imaging methods in identifying functionally relevant white matter changes that may not be visible on conventional clinical MRI. The results are in agreement with the notion that processing speed is a domain-general cognitive ability and may suggest that fast and efficient neural processing is dependent on the integrity of many tracts simultaneously.

Our findings extend previous reports of white matter injury in SCA $^{16,17}$ by highlighting possible functional consequences of such injury, and may reflect links between widespread axonal damage, demyelination, and/or disorganization of fibers and slower processing speed. It has been suggested that demyelination can be represented in DTI parameters by decreases in FA and increases in $\mathrm{RD}$ with no change in axial diffusivity, ${ }^{38}$ and in NODDI parameters by decreases in ICVF with no change in ODI. ${ }^{18,39,40}$ Our findings are consistent with this pattern. However, $\mathrm{MD}$ appeared to be the most sensitive metric, with more than double the number of voxels correlating with PSI than FA. In this sample, therefore, NODDI did not appear to offer improved sensitivity to functionally relevant microstructural changes. Rather, NODDI and DTI metrics were similarly sensitive.

Of note, the histopathologic processes that drive changes in imaging parameters are not well established, and although NODDI overcomes certain specificity issues in DTI, regions with crossing fibers remain problematic. Moreover, NODDI fixes intrinsic diffusivity to an a priori value, and there is scope to further optimize the choice of this value in the model. Before diffusion changes can confidently be referred to as markers of specific microstructural changes, further work comparing diffusion metrics not only to each other, but also to histology measures, is required.

In this sample, $44 \%$ of patients were identified with SCI. Lesions were most frequently bilateral and located in the border zones between arterial distributions in the frontal white matter. These findings are consistent with previous studies using similar MRI protocols and criteria for SCI. ${ }^{41}$ Hemoglobin was lower in patients with SCI than in patients reported radiologically as normal, but $\mathrm{SpO}_{2}$ was not. Differences in hemoglobin remained after patients on transfusion were removed from the analysis, suggesting more severe anemia in patients with SCI.

However, there were no differences in FSIQ or PSI as a function of SCI, irrespective of age. Similarly, there were no differences between patients with and without SCI in relationships between white matter microstructure parameters and processing speed, and correlated regions only partially overlapped with lesions, confirming the contribution of loss of integrity in normal-appearing white matter to reduced processing speed. These findings accord with recent studies 4,15 and, taken together, suggest that neither white matter abnormalities nor cognitive deficits are explicable solely by the presence of SCI; other factors, whether psychosocial or disease-related, are likely to be involved. 


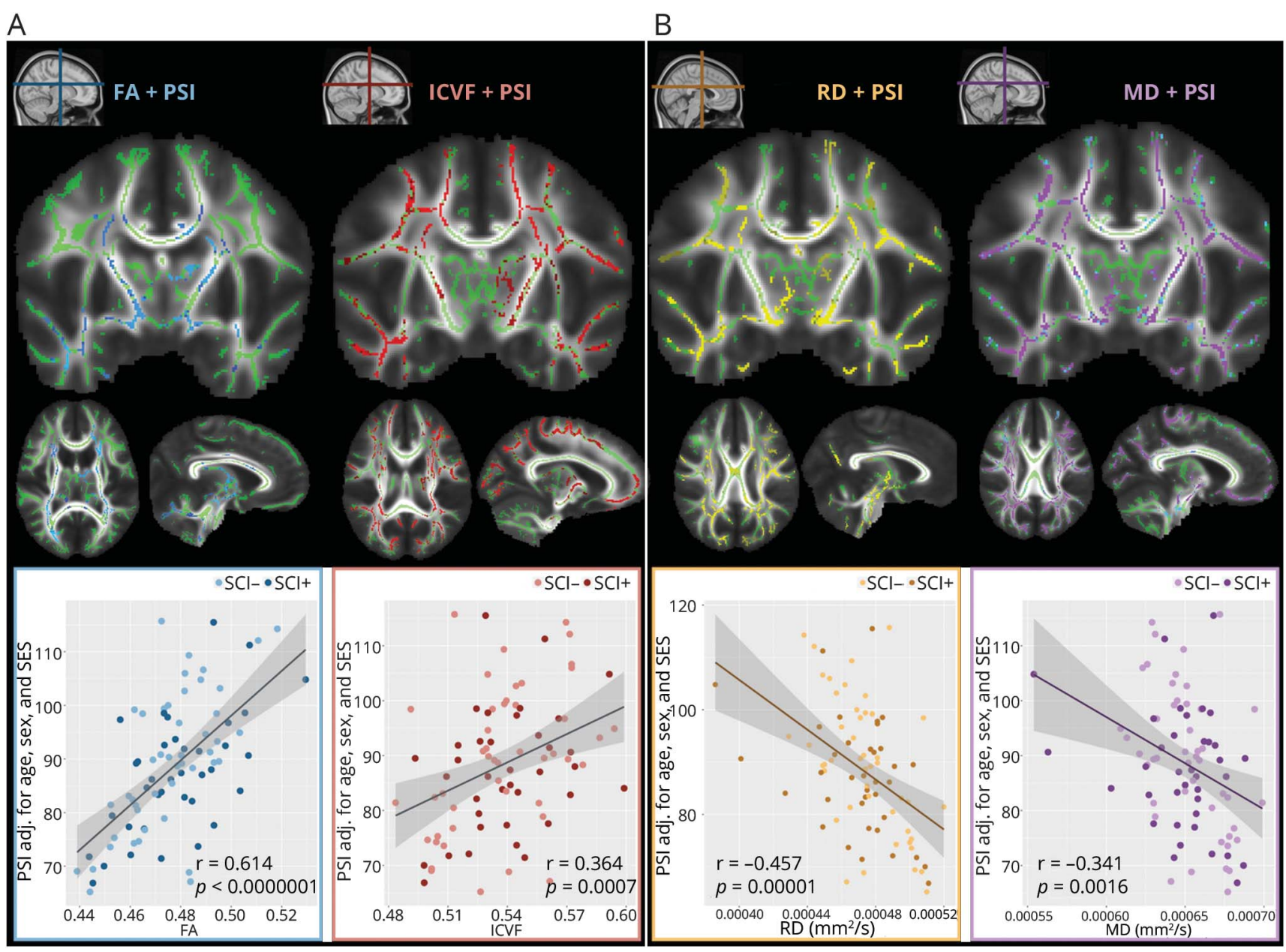

(A) Blue voxels indicate areas in which fractional anisotropy (FA) correlated with processing speed index (PSI) $(34,392$ voxels, $p<0.05)$. Red voxels indicate areas in which intracellular volume fraction (ICVF) correlated with PSI (70,659 voxels, $p<0.05)$. (B) Yellow voxels indicate areas in which radial diffusivity (RD) correlated with PSI $(67,296$ voxels, $p<0.05)$. Purple voxels indicate areas in which mean diffusivity (MD) correlated with PSI $(82,663$ voxels, $p<0.05)$. Results were age, sex, education decile (SES), and threshold-free cluster enhancement corrected and overlaid on the group white matter skeleton (green) and the study-specific mean FA template. Adj. = adjusted; SCI = silent cerebral infarction; SES = socioeconomic status.

In patients, education decile was associated with PSI, an indication that this postcode-based index was able to capture some of the variance in SES that may affect cognition. Previous research has similarly highlighted that lower maternal education may be a risk factor for slower processing speed in SCA. ${ }^{36}$ Moreover, in our regression model, education decile was identified as an independent predictor of PSI, further suggesting that socioeconomic and educational deprivation may be risk factors for slower processing speed in patients with SCA.

However, any effects of education decile were controlled for in our comparisons between patients and controls in cognition, and the control participants in this sample were siblings to patients; therefore, SES differences are unlikely to have had a major effect on the difference in PSI reported in the present study. Moreover, in our exploratory model, $\mathrm{CaO}_{2}$, age, and male sex were also identified as predictors of PSI, suggesting that there may be multiple causal pathways to cognitive deficits in SCA, in which disease and socioeconomic factors may interact.

One explanation for this pattern of results may be that SCI is associated with acute anemic events, ${ }^{42}$ where inadequate perfusion within the watershed distribution leads to infarction in brain tissue. Acute anemic events are more common in patients with lower steady-state hemoglobin, ${ }^{41}$ perhaps accounting for the relationship between anemia severity and SCI observed here. By contrast, white matter damage that is below the resolution of clinical MRI may be the result of less severe, but sustained, exposure to hypoxia ${ }^{15}$ secondary to compensatory increases in cerebral blood flow ${ }^{43}$ accompanied by reduced cerebrovascular reserve ${ }^{9}$ and increased oxygen extraction fraction. $^{7,10,11}$ This damage may be more diffuse ${ }^{15,17}$ and therefore more functionally significant, potentially explaining the presence of relationships between PSI and diffusion metrics, the absence of relationships with SCI, and the links 
between both types of damage and anemia severity. The additional identified predictors of PSI are not inconsistent with this explanation, as hydroxyurea use and recent crisis-related transfusion both increase hemoglobin and $\mathrm{SpO}_{2}$ and are prescribed more often in patients with greater disease burden, and there is evidence that males have more severe disease courses. ${ }^{41}$ These findings may explain previous discrepancies in the literature and underscore the need for researchers and clinicians to consider the interplay among risk factors as well as potential confounding effects of treatment.

The identification of $\mathrm{CaO}_{2}$ as an independent predictor of PSI is consistent with previous reports of relationships between lower $\mathrm{SpO}_{2}$ and cognitive impairment ${ }^{44}$ and white matter damage in SCA, ${ }^{15}$ and with reports of processing speed deficits in the general pediatric population with iron-deficiency anemia ${ }^{45}$ and sleep-disordered breathing ${ }^{46}$ as well as in those living at high altitude. ${ }^{47}$ Taken together, these results may suggest improvement of deficits following interventions that target hypoxemic exposure. Overnight respiratory support appears to be safe and viable in children with SCA and is a treatment option that may hold promise. ${ }^{23}$

The study utilized medical records, and there was significant between-patient variation, with time between steady-state full blood count and $\mathrm{SpO}_{2}$ measurement to cognitive assessment varying from 1 day to 6 months. There are few data on the stability of these measures over time. Although low $\mathrm{SpO}_{2}$ predicts neurologic complications in $\mathrm{SCA}^{48}$ in patients with hemoglobinopathies, right shift of the oxygen dissociation curve and the presence of carboxyhemoglobin and methemoglobin may lead to overestimation. ${ }^{49}$ Daytime and nocturnal $\mathrm{SpO}_{2}$ are not necessarily correlated in SCA, with a greater proportion of patients experiencing desaturation at night. ${ }^{50}$ Furthermore, we used postcode rather than direct measures of SES, which were unavailable for the majority. Because of these limitations, we were not able to comprehensively model specific disease and socioeconomic risk factors for slower processing speed.

Further work is required not only to determine risk factors for and mechanisms of white matter injury and cognitive impairment in SCA but also to establish whether the underlying pathology is preventable or reversible. To this end, future work will need to disentangle the effects of SCA pathology and to separate them from the effects of psychosocial factors. As this will require regression and potentially more advanced statistical modeling, future quantitative MRI studies are warranted.

This study provides evidence that reduced processing speed is correlated with widespread white matter abnormalities using quantitative microstructure parameters from multishell diffusion MRI. Although lesion status is frequently used as a proxy of disease severity, the results from this study indicate cognitive difficulties in the absence of SCI and highlight the consequences of possible damage to normal-appearing white matter. Clinicians should therefore assess for cognitive difficulties irrespective of presence of SCI, and future research should utilize diffusion MRI as a tool to further investigate potential mechanisms of cognitive impairment in SCA as well as to monitor therapies designed to ameliorate cognitive dysfunction. This study adds to a growing body of evidence indicating imaging abnormalities and cognitive impairment that may worsen with age in SCA, which together highlight the need to investigate the effect of early treatment delivery.

\section{Author contributions}

Hanne Stotesbury: literature search, study design and conception, data collection, neuropsychological assessment, data analysis, data interpretation, drafting a significant portion of the manuscript at all stages. Fenella J. Kirkham: literature search, study design and conception, data interpretation, drafting a significant portion of the manuscript at all stages. Melanie Kölbel: data collection, neuropsychological assessment, manuscript review. Philippa Balfour: data collection, neuropsychological assessment, manuscript review. Jonathan D. Clayden: data interpretation, edited second draft of manuscript, manuscript review. Sati Sahota: patient recruitment, data collection, manuscript review. Simrat Sakaria: patient recruitment, data collection, manuscript review. Dawn E. Saunders: radiologic interpretation of MRI, diagnosis of lesions, manuscript review. Jo Howard: patient recruitment, data collection, edited second draft of manuscript, manuscript review. Rachel Kesse-Adu: patient recruitment, data collection, manuscript review. Baba Inusa: patient recruitment, data collection, edited second draft of manuscript. Maria Pelidis: patient recruitment, data collection, manuscript review. Subarna Chakravorty: patient recruitment, data collection, manuscript review. David C. Rees: patient recruitment, data collection, manuscript review. Moji Awogbade: patient recruitment, data collection, manuscript review. Olu Wilkey: patient recruitment, data collection, manuscript review. Mark Layton: patient recruitment, data collection, manuscript review. Christopher A. Clark: literature search, study design and conception, data interpretation, edited second draft of manuscript, manuscript review. Jamie M. Kawadler: literature search, study design and conception, data collection, neuropsychological assessment, data interpretation, drafting a significant portion of the first draft of the manuscript, manuscript review.

\section{Acknowledgment}

The authors acknowledge the efforts of the Great Ormond Street Hospital research radiographers, without whom the study would not have been possible. The work was supported by the National Institute for Health Research Biomedical Research Centre at Great Ormond Street Hospital for Children NHS Foundation Trust and University College London.

\section{Study funding}

Ms. Stotesbury is funded by Action Medical Research (GN2509) and Dr. Kawadler is funded by Great Ormond 
Street Children's Charity (V4615) (gosh.org/what-we-do/ grant-funding/recently-funded-projects/national-calls). The National Institute for Health Research (UK; PB-PG-111229099) and National Heart, Lung, and Blood Institute (USA; R01HL079937) provided funding for recruitment, and the work was supported by the NIHR Great Ormond Street Hospital Biomedical Research Centre. Dr. Kawadler was supported by Great Ormond Street Hospital Children's Charity (national call for applications in rare disease research, V4615). The views expressed are those of the authors and not necessarily those of the NHS, the NIHR, or the Department of Health.

\section{Disclosure}

The authors report no disclosures relevant to the manuscript. Go to Neurology.org/ $\mathrm{N}$ for full disclosures.

Received December 18, 2017. Accepted in final form March 14, 2018.

\section{References}

1. Wang W, Enos L, Gallagher D, et al. Neuropsychologic performance in school-aged children with sickle cell disease: a report from the Cooperative Study of Sickle Cell Disease. J Pediatr 2001;139:391-397.

2. Ruffieux N, Njamnshi AK, Wonkam A, et al. Association between biological markers of sickle cell disease and cognitive functioning amongst Cameroonian children. Child Neuropsychol 2013;19:143-160.

3. Anie KA, Steptoe A, Bevan DH. Sickle cell disease: pain, coping and quality of life in a study of adults in the UK. Br J Health Psychol 2002;7:331-344.

4. Vichinsky EP, Neumayr LD, Gold JI, et al. Neuropsychological dysfunction and neuroimaging abnormalities in neurologically intact adults with sickle cell anemia. JAMA 2010;303:1823-1831.

5. Crawford RD, Jonassaint CR. Adults with sickle cell disease may perform cognitive tests as well as controls when processing speed is taken into account: a preliminary case-control study. J Adv Nurs 2016;72:1409-1416.

6. Helton KJ, Paydar A, Glass J, et al. Arterial spin-labeled perfusion combined with segmentation techniques to evaluate cerebral blood flow in white and gray matter of children with sickle cell anemia. Pediatr Blood Cancer 2009;52:85-91.

7. Fields ME, Guilliams KP, Ragan DK, et al. Regional oxygen extraction predicts border zone vulnerability to stroke in sickle cell disease. Neurology 2018;90:e1134-e1142.

8. Leung J, Duffin J, Fisher JA, Kassner A. MRI-based cerebrovascular reactivity using transfer function analysis reveals temporal group differences between patients with sickle cell disease and healthy controls. Neuroimage Clin 2016;12:624-630.

9. Kosinski PD, Croal PL, Leung J, et al. The severity of anaemia depletes cerebrovascular dilatory reserve in children with sickle cell disease: a quantitative magnetic resonance imaging study. Br J Haematol 2017;176:280-287.

10. Jordan LC, Gindville MC, Scott AO, et al. Non-invasive imaging of oxygen extraction fraction in adults with sickle cell anaemia. Brain 2016;139:738-750.

11. Watchmaker JM, Juttukonda MR, Davis LT, et al. Hemodynamic mechanisms underlying elevated oxygen extraction fraction (OEF) in moyamoya and sickle cell anemia patients. J Cereb Blood Flow Metab Epub 2016 Jan 1.

12. Mackin RS, Insel P, Truran D, et al. Neuroimaging abnormalities in adults with sickle cell anemia: associations with cognition. Neurology 2014;82:835-841.

13. Scantlebury N, Mabbott D, Janzen L, et al. White matter integrity and core cognitive function in children diagnosed with sickle cell disease. J Pediatr Hematol Oncol 2011. 33:163-171.

14. Choi S, Bush AM, Borzage MT, et al. Hemoglobin and mean platelet volume predicts diffuse T1-MRI white matter volume decrease in sickle cell disease patients. Neuroimage Clin 2017;15:239-246.

15. Kawadler JM, Kirkham FJ, Clayden JD, et al. White matter damage relates to oxygen saturation in children with sickle cell anemia without silent cerebral infarcts. Stroke 2015;46:1793-1799.

16. Balci A, Karazincir S, Beyoglu Y, et al. Quantitative brain diffusion-tensor MRI findings in patients with sickle cell disease. AJR Am J Roentgenol 2012;198: 1167-1174.

17. Sun B, Brown RC, Hayes L, et al. White matter damage in asymptomatic patients with sickle cell anemia: screening with diffusion tensor imaging. AJNR Am J Neuroradiol 2012;33:2043-2049.

18. Zhang H, Schneider T, Wheeler-Kingshott CA, Alexander DC. NODDI: practical in vivo neurite orientation dispersion and density imaging of the human brain. Neuroimage 2012;61:1000-1016.
19. Chevalier N, Kurth S, Doucette MR, et al. Myelination is associated with processing speed in early childhood: preliminary insights. PLoS One 2015;10:e0139897.

20. Chopra S, Shaw M, Shaw T, Sachdev PS, Anstey KJ, Cherbuin N. More highly myelinated white matter tracts are associated with faster processing speed in healthy adults. Neuroimage 2018;171:332-340.

21. Chang YS, Owen JP, Pojman NJ, et al. White matter changes of neurite density and fiber orientation dispersion during human brain maturation. PLoS One 2015;10: e0123656.

22. Rosen CL, Debaun MR, Strunk RC, et al. Obstructive sleep apnea and sickle cell anemia. Pediatrics 2014;134:273-281.

23. Howard J, Slee AE, Skene S, et al. Overnight auto-adjusting continuous airway pressure + standard care compared with standard care alone in the prevention of morbidity in sickle cell disease: protocol for a phase II randomised controlled tria (POMS2B). Trials 2018;19:55.

24. Williams PE, Weiss LG, Rolfhus EL. WISC-IV Technical Report \#2: Psychometric Properties. San Antonio: Psychological Corp; 2003:1-6.

25. McCrimmon AW, Smith AD. Review of the Wechsler Abbreviated Scale of Intelligence, Second Edition (WASI-II) Wechsler D. (2011). J Psychoeduc Assess 2013; 31:337-341.

26. DCLG. The English indices of deprivation 2010. Neighb Stat Release 2011:1-20.

27. DeBaun MR, Gordon M, McKinstry RC, et al. Controlled trial of transfusions for silent cerebral infarcts in sickle cell anemia. N Engl J Med 2014;371:699-710.

28. Clayden JD, Muñoz Maniega S, Storkey AJ, King MD, Bastin ME, Clark CA. TractoR magnetic resonance imaging and tractography with R. J Stat Softw 2011;44:1-18

29. Jenkinson M, Beckmann CF, Behrens TEJ, Woolrich MW, Smith SM. FSL. Neuroimage 2012;62:782-790.

30. Smith SM, Jenkinson M, Johansen-Berg H, et al. Tract-based spatial statistics: voxelwise analysis of multi-subject diffusion data. Neuroimage 2006;31:1487-1505.

31. Hua K, Zhang J, Wakana S, et al. Tract probability maps in stereotaxic spaces: analyses of white matter anatomy and tract-specific quantification. Neuroimage 2008;39: 336-347.

32. Fox J, Weisberg S. An R Companion to Applied Regression, 2nd ed. Thousand Oaks: Sage Publications; 2011.

33. Peña EA, Slate EH. Global validation of linear model assumptions. J Am Stat Assoc 2006;101:341-354.

34. Harvey PD. Clinical applications of neuropsychological assessment. Dialogues Clin Neurosci 2012;14:91-99.

35. Jung RE, Haier RJ. The Parieto-Frontal Integration Theory (P-FIT) of intelligence: converging neuroimaging evidence. Behav Brain Sci 2007;30:135-154.

36. Oluwole OB, Noll RB, Winger DG, Akinyanju O, Novelli EM. Cognitive functioning in children from Nigeria with sickle cell anemia. Pediatr Blood Cancer 2016;63: 1990-1997.

37. Marchman VA, Fernald A. Speed of word recognition and vocabulary knowledge in infancy predict cognitive and language outcomes in later childhood. Dev Sci 2008;11: F9-F16.

38. Aung WY, Mar S, Benzinger TL. Diffusion tensor MRI as a biomarker in axonal and myelin damage. Imaging Med 2013;5:427-440.

39. Kipp L, Cawley N, Prados F, et al. Neurite orientation dispersion and density imaging (NODDI) in RRMS (P4.159). Neurology 2016;86:P4.159.

40. Jespersen SN, Bjarkam CR, Nyengaard JR, et al. Neurite density from magnetic resonance diffusion measurements at ultrahigh field: comparison with light microscopy and electron microscopy. Neuroimage 2010;49:205-216.

41. DeBaun MR, Armstrong FD, McKinstry RC, Ware RE, Vichinsky E, Kirkham FJ. Silent cerebral infarcts: a review on a prevalent and progressive cause of neurologic injury in sickle cell anemia. Blood 2012;119:4587-4596.

42. Dowling MM, Quinn CT, Plumb P, et al. Acute silent cerebral ischemia and infarction during acute anemia in children with and without sickle cell disease. Blood Am Soc Hematol 2012;120:3891-3897.

43. Bush AM, Borzage MT, Choi S, Vaclavu L. Determinants of resting cerebral blood flow in sickle cell disease. Am J Hematol 2016;91:912-917.

44. Hollocks MJ, Kok TB, Kirkham FJ, et al. Nocturnal oxygen desaturation and disordered sleep as a potential factor in executive dysfunction in sickle cell anemia. J Int Neuropsychol Soc 2012;18:186-173.

45. Murray-Kolb LE, Beard JL. Iron treatment normalizes cognitive functioning in young women. Am J Clin Nutr 2007;85:778-787.

46. Hill CM, Hogan AM, Onugha N, et al. Increased cerebral blood flow velocity in children with mild sleep-disordered breathing: a possible association with abnormal neuropsychological function. Pediatrics 2006;118:e1100-e1108.

47. Bass JL, Corwin M, Gozal D, et al. The effect of chronic or intermittent hypoxia on cognition in childhood: a review of the evidence. Pediatrics 2004;114:805-816.

48. Kirkham FJ, Hewes DKM, Prengler M, Wade A, Lane R, Evans JPM. Nocturnal hypoxaemia and central-nervous-system events in sickle-cell disease. Lancet 2001, $357: 1656-1659$

49. Blaisdell CJ, Goodman S, Clark K, Casella JF, Loughlin GM. Pulse oximetry is a poor predictor of hypoxemia in stable children with sickle cell disease. Arch Pediatr Adolesc Med 2000; 154:900-903.

50. Halphen I, Elie C, Brousse V, et al. Severe nocturnal and postexercise hypoxia in children and adolescents with sickle cell disease. PLoS One 2014;9:e97462. 


\section{Neurology}

\section{White matter integrity and processing speed in sickle cell anemia \\ Hanne Stotesbury, Fenella J. Kirkham, Melanie Kölbel, et al. \\ Neurology 2018;90;e2042-e2050 Published Online before print May 11, 2018 \\ DOI 10.1212/WNL.0000000000005644}

\section{This information is current as of May 11, 2018}

\section{Updated Information \& Services}

\section{References}

Citations

Subspecialty Collections

Permissions \& Licensing

Reprints including high resolution figures, can be found at: http://n.neurology.org/content/90/23/e2042.full

This article cites 46 articles, 6 of which you can access for free at: http://n.neurology.org/content/90/23/e2042.full\#ref-list-1

This article has been cited by 1 HighWire-hosted articles: http://n.neurology.org/content/90/23/e2042.full\#\#otherarticles

This article, along with others on similar topics, appears in the following collection(s):

\section{Hematologic}

http://n.neurology.org/cgi/collection/hematologic

Infarction

http://n.neurology.org/cgi/collection/infarction

Intelligence

http://n.neurology.org/cgi/collection/intelligence

Information about reproducing this article in parts (figures,tables) or in its entirety can be found online at:

http://www.neurology.org/about/about_the_journal\#permissions

Information about ordering reprints can be found online:

http://n.neurology.org/subscribers/advertise

Neurology ${ }^{\circledR}$ is the official journal of the American Academy of Neurology. Published continuously since 1951, it is now a weekly with 48 issues per year. Copyright Copyright ( 2018 The Author(s). Published by Wolters Kluwer Health, Inc. on behalf of the American Academy of Neurology.. All rights reserved. Print ISSN: 0028-3878. Online ISSN: 1526-632X.

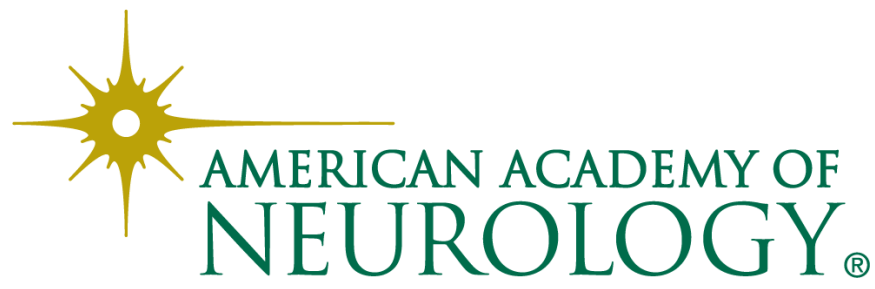

